\title{
Establishment of a national focal point to monitor the Performance of PCR Centres in Sri Lanka
}

Athapattu P, Wijenayake PH, Weediyawatta PKGP.

\begin{abstract}
Introduction: The number of confirmed cases of COVID -19 informs us about the development and magnitude of the global pandemic. Confirmation of COVID-19 infection is mainly by testing with PCR. The necessity of evaluating the performance of PCR laboratories was identified to understand how the pandemic was progressing, how to respond appropriately to the threat; either as individuals or as a society, to learn where countermeasures against the pandemic worked and for planning. Reliable data on testing was therefore necessary to assess spread of the pandemic.
\end{abstract}

Objective: To establish a national focal point to collect quality, accurate, timely data of PCR test performance for planning and to make decision.

Methods: Defining of data elements for collection and monitoring of the performance of PCR laboratories was done as the first step. Digital solution was identified after assessing the requirement. Agile development method was used to develop the digital solution, which consists of five modules. Entered data on PCR performance of laboratories were analyzed by the officer in charge of the focal point and disseminated timely, accurate, complete and compile data to the relevant officials on daily basis for further planning and implementation.

Results: Performance of individual laboratories was identified on daily basis. Private sector performance was higher than public sector. Peaks showed the local clusters.

Conclusion: A quality, accurate and real-time data and information helped for remarkable improvement in every step of planning and decision making to overcome the global challenge of COVID-19.

Limitations: Computer literacy, close monitoring and duplication of data affected for accuracy.

Key Words: COVID-19, PCR Test, Performance of laboratories

\section{Introduction}

COVID 19- infection has spread as pandemic form throughout the world. Sri Lanka too is significantly affected by this pandemic. The World Health Organization (WHO) declared the COVID-19 infection as a Public Health Emergency of International Concern (1).

The establishment of the presidential task force and enacting several regulations under the Quarantine and Prevention of Diseases Ordinance (1897) were initial COVID-19 mitigation measures of Sri Lanka (2). The index case of Sri Lanka, a Chinese tourist was detected on the 27th of January. The first indigenous patient was diagnosed on the 11th of March. The government took immediate measures, and the country has been kept in lockdown mode to prevent the spread of the disease in Sri Lanka (3).

WHO declared the RT-PCR test as the confirmatory laboratory test (Gold standard test), which Sri Lanka adopted initially (4-7). With the gradual increase of cases, the need for accurate, reliable and real-time data on laboratories' performance was identified. That information was used to understand the pandemic, determine which outcomes should be prioritized, plan resource allocation, decide intervention of health care delivery, and compare meaningfully with other countries (8). Because of the rapidly changing and uncertain context of the pandemic, it was essential to build up feedback loops and frequent pause points to revisit how things are happening in the field. The data collection, storage, processing, and dissemination via online systems would be more beneficial as access to the laboratories was limited during the pandemic (9).

The Director General of Health Services (DGHS), the competent authority, ordered to establish a National Focal Point to gather daily performance of PCR test at the laboratories. The Directorate of Primary Care services, Ministry of Health $(\mathrm{MOH})$ was assigned this task to collect quality, accurate, real-time data on the performance of PCR laboratories and compile, analysis and dissemination of information efficiently and effectively since the end of March 2020. 


\section{2) Objectives}

2.1 General Objective

To establish a national focal point to monitor the performance of PCR test Centers in Sri Lanka.

2.2 Specific objectives

- To design a user friendly, comprehensive, online operational data base

- To identify and trained focal point from each functioning lab

- To maintain timelines, accuracy and completeness of data

- To compile and disseminate a daily report for decision makers and planners

\section{Methodology}

Ten data elements were defined (Table 1) to monitor the performance of PCR laboratories by obtaining inputs from relevant stakeholders.

Table 1: Data elements and definitions

\begin{tabular}{|c|c|}
\hline No Data Element & Definition \\
\hline 1 Name of the Laboratory & The laboratory where the sample was tested \\
\hline 2 Sample Received From & The place from where the sample was sent to the lab \\
\hline 3 Number of Samples Received - First Time Patients & $\begin{array}{l}\text { Sample received from a patient who was suspected to } \\
\text { having COVID- } 19 \text {. }\end{array}$ \\
\hline 4 Number of Samples Received - Repeat (Patients) & $\begin{array}{l}\text { Sample received from a patient who was already } \\
\text { diagnosed and having treatment for COVID-19 }\end{array}$ \\
\hline $5 \quad$ Number of Samples Tested - First Time Patients & $\begin{array}{l}\text { Number of samples tested out of "Samples Received - } \\
\text { First Time Patients" }\end{array}$ \\
\hline 6 Number of Samples Tested - Repeat (Patients) & $\begin{array}{l}\text { Number of samples tested out of "Samples Received - } \\
\text { Repeat (Patients)" }\end{array}$ \\
\hline 7 Number of Positive Samples - First Time & $\begin{array}{l}\text { Number of positive samples out of "Samples Tested - } \\
\text { First Time Patients" }\end{array}$ \\
\hline 8 Number of Positive Samples - Repeat Patient & $\begin{array}{l}\text { Number of positive samples out of "Samples Tested - } \\
\text { Repeat Patients" }\end{array}$ \\
\hline 9 Inconclusive Samples & No of samples tested and unable to give result \\
\hline 10 Invalid Samples & $\begin{array}{l}\text { Contaminated samples due to various reasons from } \\
\text { the time of sample collection to sample testing }\end{array}$ \\
\hline
\end{tabular}

Requirements for digital solution were identified via key stakeholder interviews. Rapid literature review and market analysis were conducted to find a suitable product (10-15). The agile development method was used to develop the digital solution. It consists of an online data entry module for collecting aggregated data, a central database, a realtime data visualization module, a data analytic module and a report module. Initial development and deployment completed within 2 days with zero development cost. Online user training was given to the end-users (pre-identified officer at the laboratory), and an electronic comprehensive user manual was provided. Also, round the clock user support was given to improve data quality and to maintain the sustainability of the digital solution.

\section{Process}

The prescribed time for data entry of daily performance was before $10.00 \mathrm{pm}$ daily, but data entered up to midnight was considered for the final report. SMS reminders were sent to the laboratories to enter data by $10.00 \mathrm{pm}$ that ensured completeness. The compiled daily performance report was disseminated to the heads of the laboratory and institutions daily at 7.00 am of the following day to get feedback and cross-check the accuracy. 
PCR laboratories initiated entering the data since 24th March 2020 into the system. The paper based data collected from 2020.02.18 were entered by the National Focal Point. Initially there were 6 labs by late March which increased up to 25 laboratories by the end of September 2020 and 36 laboratories identified by the end of 28th February 2021.

\section{Results}

Compilation and data analysis was carried out at the national focal point, and the chief controlling officer sent an interim report to Intelligence Services (SIS) and the National Operation Centre for Prevention of COVID - 19 Outbreak every day at $10 \mathrm{pm}$. The final report was prepared before $7.00 \mathrm{am}$ of the following day and sent to SIS, National Operational Centre, Ministry Officials, all consultants in charge of laboratories and heads of the institutions to assess their own performances and for data validation with their feedback.

Table 2 indicates the samples tested during the reporting period $(2020.02 .18-2021.02 .28)$

Table 2: Summary of PCR performance.

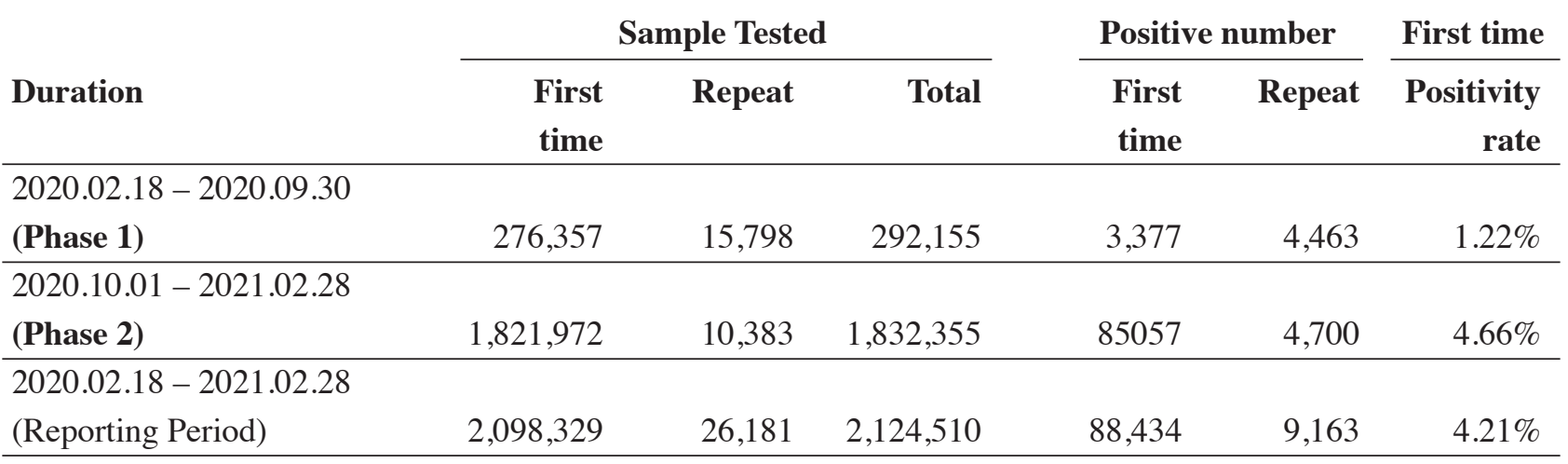

Note 1: Percentage First-time positivity rate $=($ First-time positive/Sample tested in first-time*100)

During phase 1, the total number of samples tested was 292,155 and out of them 276,357 (94.59\%) were suspected (First time) patients and 15,798 (5.41\%) tests were conducted on diagnosed (Repeat) patients who were at treatment centres. The positivity rate of first time was $1.22 \%(3,377 / 276,357)$.

During phase 2, Total number of samples tested was 1,832,355 and out of them 1,821,972(99.43\%) samples were tested in first time patients, $10,383(0.57 \%)$ tested on diagnosed patients who were at treatment centres. The positivity rate of first time was $4.66 \%(850571 / 832,355)$.

Total number of samples tested during the reporting period was 2,124,510 and out of them 2,098,329 (98.76\%), samples were tested in suspected patients and 26,181 (1.24\%) tests conducted in diagnosed patients. The positivity rate of reporting period was $4.21 \%(88,434 / 2,098,329)$.

Daily and cumulative report when required was issued as per the details in table 3 . Also daily average, district wise community samples tested, and private sector performance report also issued as a routine. 
The performance of individual laboratories are described in table 3.

Table 3: Performance of Individual Laboratories

\begin{tabular}{|c|c|c|c|c|c|}
\hline \multirow[b]{2}{*}{ Laboratory Name } & \multicolumn{2}{|c|}{ Samples } & \multicolumn{2}{|c|}{ Positive Samples } & \multirow{2}{*}{$\begin{array}{c}\text { Samples } \\
\text { Inconclusive }\end{array}$} \\
\hline & Received & Tested & First Time & Repeat & \\
\hline Medical Research Institute (MRI) & 236579 & 227990 & 10432 & 752 & 1616 \\
\hline BIA - Katunayake & 205897 & 205693 & 10396 & 128 & 2562 \\
\hline TH - Karapitiya & 135341 & 135483 & 4430 & 1027 & 784 \\
\hline BH-Colombo East (Mulleriyawa) & 115538 & 112339 & 8535 & 18 & 3397 \\
\hline National Hospital - Kandy & 102253 & 102325 & 4797 & 637 & 1900 \\
\hline NIID Angoda & 101604 & 101001 & 5866 & 3813 & 2375 \\
\hline TH - Anuradhapura & 86204 & 86064 & 2071 & 1243 & 1748 \\
\hline TH - Jaffna & 59203 & 59208 & 825 & 38 & 1212 \\
\hline TH - Batticoloa & 55134 & 54675 & 1952 & 287 & 313 \\
\hline PGH - Badulla & 41273 & 40859 & 1482 & 70 & 268 \\
\hline Apeksha Hospital - Maharagama & 35699 & 35934 & 1418 & 157 & 1234 \\
\hline CNTH- (Ragama) & 33577 & 33636 & 1560 & 114 & 1062 \\
\hline TH - Sri Jayawardhanapura & 26134 & 26432 & 997 & 0 & 415 \\
\hline DGH - Nuwara Eliya & 25985 & 25923 & 1246 & 2 & 349 \\
\hline TH - Rathnapura & 25484 & 25314 & 2130 & 93 & 813 \\
\hline $\mathrm{TH}$ - Kurunegala & 9060 & 9031 & 532 & 9 & 48 \\
\hline CSTH - Kalubowila & 4011 & 3872 & 479 & 0 & 56 \\
\hline National Hospital - Sri Lanka & 4887 & 4887 & 197 & 0 & 192 \\
\hline DGH - Kegalle & 1175 & 975 & 91 & 0 & 2 \\
\hline Sri Lanka Army Hospital & 32174 & 32224 & 2168 & 147 & 251 \\
\hline \multicolumn{6}{|l|}{ Private Laboratories } \\
\hline Nawaloka Hospital (pvt) Ltd & 293662 & 293588 & 6660 & 1 & 20 \\
\hline Asiri Genetic Lab & 107240 & 106449 & 2911 & 2 & 40 \\
\hline Durdans Hospital & 96315 & 96330 & 1204 & 9 & 0 \\
\hline Lanka Hospital (pvt) Ltd & 92492 & 91038 & 2067 & 5 & 6 \\
\hline Genelabs Medical (pvt) Ltd & 33445 & 33437 & 915 & 5 & 29 \\
\hline Credence Genomics (Pvt) Ltd & 4767 & 4751 & 281 & 4 & 2 \\
\hline Melsta Laboratories & 3498 & 3498 & 140 & 0 & 0 \\
\hline Hemas Hospitals & 1518 & 1518 & 35 & 0 & 0 \\
\hline Forte Diagnostics (pvt) Ltd & 1069 & 1068 & 71 & 2 & 17 \\
\hline \multicolumn{6}{|l|}{ University Laboratories } \\
\hline KDU & 30451 & 30372 & 1085 & 309 & 884 \\
\hline Faculty of Medicine - Japura & 109907 & 109818 & 10258 & 231 & 981 \\
\hline Faculty of Medicine - Jaffna & 15905 & 15711 & 324 & 1 & 19 \\
\hline Faculty of Medicine - Colombo & 6103 & 6032 & 265 & 3 & 87 \\
\hline Faculty of Medicine - Peradeniya & 3973 & 4000 & 442 & 10 & 98 \\
\hline University of Peradeniya & 1814 & 1800 & 153 & 0 & 7 \\
\hline Faculty of Medicine - Karapitiya & 1356 & 1235 & 19 & 46 & 33 \\
\hline Total & 2140727 & 2124510 & 88434 & 9163 & 22820 \\
\hline
\end{tabular}


Figure 1: Daily Average number of PCR Tests - Sri Lanka

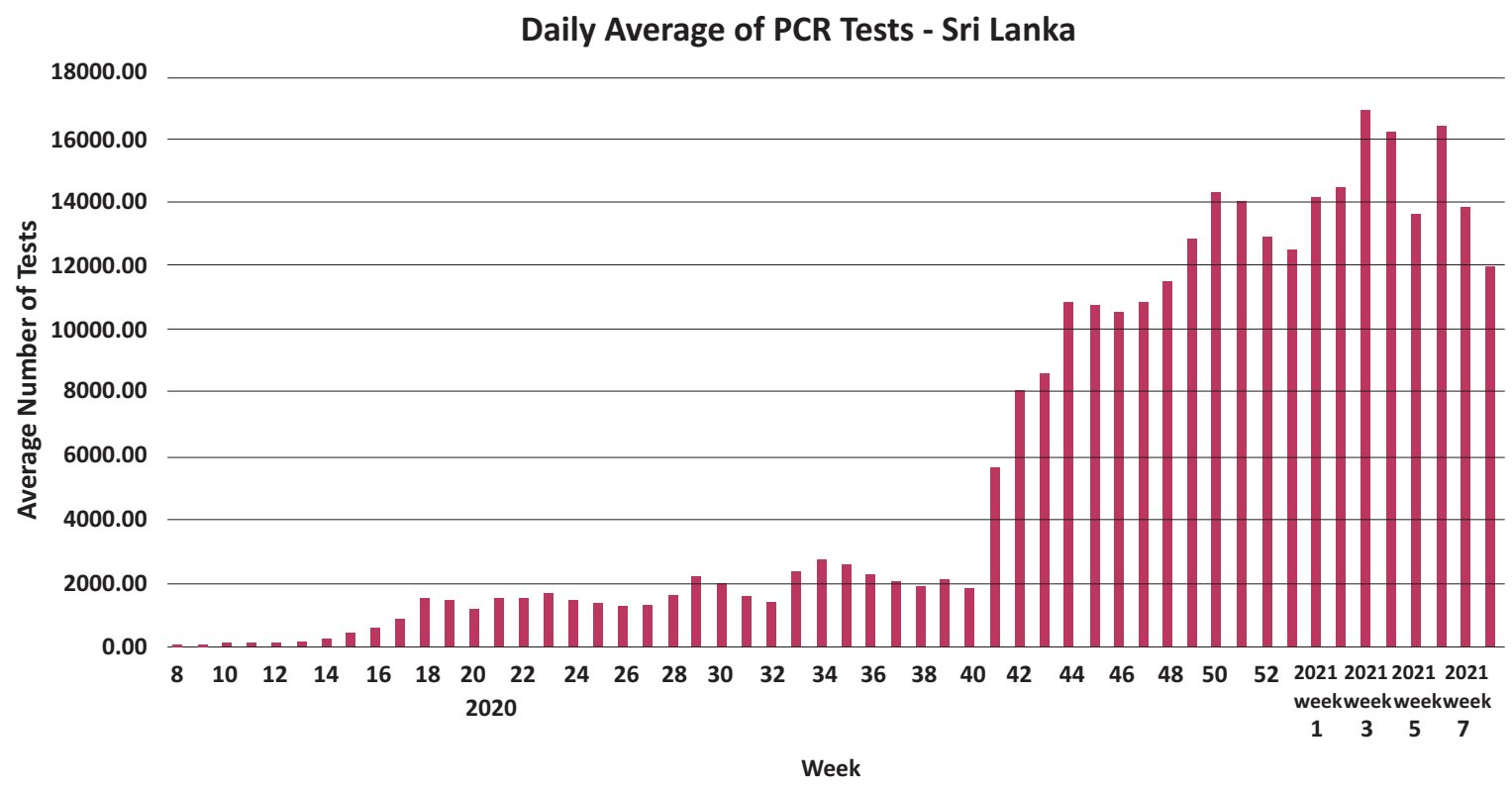

There were 21 government sector laboratories, 6 university laboratories and 9 private sector laboratories. The maximum number of tests were done by Nawaloka Hospital $(n=293588)$. The MRI and BIA tested more than 200,000 samples during the referring period out of the state sector institutions.

The average number of PCR tests conducted by the labs was calculated weekly (defined according to ISO standard). In Phase 1 (8 to 40 weeks of 2020), the maximum number was 2723 , which was on the 34 th week of 2020. In phase 2 , the maximum daily average of 16921 was achieved in the 3rd week of 2021.

The total community samples tested in reported period were $1005763(47.3 \%)$ and out of that 51923 were positive, with a rate of $5.16 \%$. During phase 1,81376 $(27.85 \%)$ samples were tested, and $424(0.52 \%)$ were positive. In phase 2, $924387(50.73 \%)$ community samples were tested, and 51499(5.57\%) were positive. In both phases, Colombo, Kaluthara and Gampaha RDHS areas reported the highest numbers for community samples.

Four private laboratories (Asiri Genetic lab, Durdans hospitals, Lanka Hospitals and Nawaloka hospitals) conducted the PCR tests in phase 1. Though it was expanded up to 10 laboratories in phase 2, only 9 laboratories entered data during the reporting period. Overall, private sector contribution was $29.73 \%$ $(n=631677)$. The majority were performed by the Nawaloka hospital during both phases.

\section{Discussion}

As the pandemic progressed over time, large-scale testing and contact tracing were the central efforts to understand the spread and respond appropriately. Based on the evidence, patient management criteria were advanced, testing of discharged patients was decided with reference to initial viral load; thus, conducting two consecutive negative samples was omitted. Therefore, the number of tests performed on diagnosed patients were reduced [Table: 02$]$.

With increased demand, accredited private sector labs were recruited to conduct PCR tests. Nawaloka hospital performance was higher than even the MRI, the reference lab that initiated in January 2020. Convenience, to maintain privacy and as positivity attached stigma [Table:03] may be the causes. Increasing testing capacity reduced the unnecessary self-isolation, depletion of workforce especially for essential services and the missing potentiality of individual cases that minimize the risk of transmission.

Introducing mass testing for the community on both symptomatic and asymptomatic cases reduced the risk of nosocomial transmission. Another key epidemiological parameter that could inform the intensity and range of social distancing strategies to combat COVID-19 is the asymptomatic proportion. Indeed, the asymptomatic proportion is a useful quantity to gauge the true burden of the disease and better interpret estimates of the transmission potential (16). The remarkable improvement was achieved in this aspect in phase two than in phase one. 
Regular feedback sessions were made for updating knowledge and technical modifications. Issues and opposing events were discussed at review meetings, technical advisory meetings and lab meetings to strengthen the process. Frequent feedbacks and daily performance reports helped to manage the continuous logistic supply. Cumulative reports were issued at different milestones. This daily performance report was validated with the comments of expert officials. Accordingly, the ministry expanded the services.

There was a difference noted between the sample tested, and the sample received in some labs. Reasons behind were the number of receiving samples was not compatible with the number of actual samples in the container, single samples tested several times in the same laboratory (duplicate sample), invalid samples and data entry errors (duplicate values).

A discrepancy was noted between figures of this system and the epidemiology unit because, the system reported number of positive samples while the epidemiology unit reported positive patients during reported period. Duplicate samples, several samples of one individual were sent to several labs, receiving multiple samples of one individual to the laboratory especially in postmortem samples were other reasons for the discrepancy.

Also, data disseminated to the Health Promotion Bureau for the national dashboard, which used for country comparison.

\section{Limitations}

Computer literacy of the data entry operator, supervision of the head of the institutions and national focal point affected the accuracy of the data. Although the system is compatible with mobile data entry, poor network connections still affect the data entry. The available data has limited value on epidemiological or demographic analysis. Strict discipline, responsibility should be maintained as individual users can enter the data for multiple laboratories.

\section{Conclusion}

Established focal point under the direction of DGHS provided remarkable improvement for accurate, effective data dissemination, maintaining appropriate logistic supply for labs to increase PCR testing capacity, project disease trend, improve patient care facilities, and identify the disease burden and for evidence-based distribution of samples among labs that reduced the overburden. Even today, this is a valuable source of information for policy planners and decision makers.

\section{References}

1. Coronavirus Disease (COVID-19) - events as they happen [Internet]. [cited 2020 Jun 23]. Available from: https://www.who.int/emergencies/diseases/ novel-coronavirus-2019/events-as-they-happen

2. Quarantine and Prevention of Diseases Ordinance [Internet]. 3 of 1897 Sep 2, 1987. Available from: https://www.quarantine.health.gov.lk/images/ pdf/q_act.pdf

3. Wickramaarachchi WPTM, Perera SSN, Jayasinghe S. COVID-19 Epidemic in Sri Lanka: A Mathematical and Computational Modelling Approach to Control. Blyuss K, editor. Comput Math Methods Med. 2020 Oct 19;2020:4045064.

4. LABORATORY STRATEGY FOR COVID-19 IN SRI LANKA.pdf [Internet]. [cited $2021 \mathrm{Mar}$ 15]. Available from: https://www.who.int/docs/ default-source/searo/sri-lanka/lab-strategy-finalcompressed.pdf?sfvrsn=dc3ffeca_0

5. Diagnostic testing for SARS-CoV-2 [Internet]. [cited 2021 Mar 15]. Available from: https://www. who.int/publications/i/item/diagnostic-testing-forsars-cov-2

6. Goudouris ES. Laboratory diagnosis of COVID-19. J Pediatr (Rio J). 2021;97(1):7-12.

7. Epidemiology Unit of The Ministry of Health and, Indigenous Medical Services. Covid-19 Laboratory Test Strategy in Sri Lanka Version II [Internet]. Ministry of Health, Sri Lanka; 2020. Available from: https://www.epid.gov.lk/web/images/pdf/ Circulars/Corona_virus/f inal_draft_of_testing _ strategy_v_2.pdf

8. Organization WH. Coronavirus disease 2019 ( $\square$ COVID-19) $\square$ : situation report, 94. World Health Organization; 2020 Apr p. 12 p.

9. Ziegler J, Mason P. Adapting data collection and utilisation to a Covid-19 reality. :11.

10. Kumar SM, Belwal M. Performance dashboard: Cutting-edge business intelligence and data visualization. In: 2017 International Conference On Smart Technologies For Smart Nation (SmartTechCon). 2017. p. 1201-7. 
11. Toasa R, Maximiano M, Reis C, Guevara D. Data visualization techniques for real-time information \#x2014; A custom and dynamic dashboard for analyzing surveys' results. In: 2018 13th Iberian Conference on Information Systems and Technologies (CISTI). 2018. p. 1-7.

12. Nutley T, Reynolds HW. Improving the use of health data for health system strengthening. Glob Health Action [Internet]. 2013 Feb 13 [cited 2018 Jun 11];6. Available from: https://www.ncbi.nlm. nih.gov/pmc/articles/PMC3573178/

13. Pricing \&amp; Product Comparison I Microsoft Power BI [Internet]. [cited $2020 \mathrm{Jul}$ 2]. Available from: https://powerbi.microsoft.com/en-us/pricing/

14. Data Studio [Internet]. Google Developers. [cited 2021 Mar 15]. Available from: https://developers. google.com/datastudio

15. Dolezel D, McLeod A. Big Data Analytics in Healthcare: Investigating the Diffusion of Innovation. Perspect Health Inf Manag [Internet]. 2019 Jul 1 [cited 2021 Mar 15];16(Summer). Available from: https://www.ncbi.nlm.nih.gov/ pmc/articles/PMC6669368/

16. Mizumoto K, Kagaya K, Zarebski A, Chowell G. Estimating the asymptomatic proportion of coronavirus disease 2019 (COVID-19) cases on board the Diamond Princess cruise ship, Yokohama, Japan, 2020. Eurosurveillance [Internet]. 2020 Mar 12 [cited 2021 Mar 15];25(10). Available from: https://www.ncbi.nlm.nih.gov/pmc/articles/ PMC7078829/ 